\title{
Conway names, the simplicity hierarchy and the surreal number tree
}

\author{
PHILIP EHRLICH
}

\begin{abstract}
Each surreal number has a unique Conway name (or normal form) that is characteristic of its individual properties. The paper answers the following two questions that are naturally suggested by the surreal number system's structure as a lexicographically ordered full binary tree. (i) Given the Conway name of a surreal number, what are the Conway names of its two immediate successors? (ii) Given the Conway names of the members of a chain of surreal numbers of limit length, what is the Conway name of the immediate successor of the chain?
\end{abstract}

2000 Mathematics Subject Classification 06.A05, 06A.06, 06F.25, $12 . J 15$ (primary); 03E10, $03 \mathrm{H} 05$ (secondary)

Keywords: surreal numbers, ordered binary trees, divisible ordered abelian groups, real-closed ordered fields

\section{Introduction}

In his groundbreaking investigation On Numbers and Games [4], J. H. Conway introduced a real-closed field embracing the reals and the ordinals as well as a great many less familiar numbers including $-\omega, \omega / 2,1 / \omega, \sqrt{\omega}$ and $\omega-\pi$, to name only a few. Indeed, this particular real-closed field, which Conway's calls No, is so remarkably inclusive that, subject to the proviso that numbers-construed here as members of ordered "number" fields - be individually definable in terms of sets of von NeumannBernays-Gödel set theory with global choice (NBG) [16], it may be said to contain "All Numbers Great and Small." In this respect, Conway's ordered field No of surreal numbers bears much the same relation to ordered fields that the ordered field $\mathbb{R}$ of real numbers bears to Archimedean ordered fields [9]; also see [8], [11, page 1231].

In addition to its inclusive structure as an ordered field, No has a rich hierarchical structure that emerges from the recursive clauses in terms of which it is defined. From the standpoint of Conway's construction, this algebraico-tree-theoretic structure, or simplicity hierarchical structure, as we call it [11], [10], depends upon No's implicit 
structure as a lexicographically ordered binary tree and arises from the fact that the sums and products of any two members of the tree are the simplest possible elements of the tree consistent with No's structure as an ordered group and an ordered field, respectively, it being understood that $x$ is simpler than $y$ just in case $x$ is a predecessor of $y$ in the tree.

In the author's [10], [12] and [13], the just-described simplicity hierarchy was brought to the fore and made part of an algebraico-tree-theoretic definition of No. In [11] we introduced a novel class of structures whose properties generalize those of No thus construed and explored some of the relations that exist between No and this more general class of s-hierarchical ordered structures. In particular, we defined a number of types of s-hierarchical ordered structures (groups, fields, vector spaces, etc.) as well as a corresponding type of $s$-hierarchical mapping, identified No as a complete s-hierarchical ordered group (s-hierarchical ordered field, etc.), and showed that there is one and only one s-hierarchical mapping of an s-hierarchical ordered structure into No. These mappings were found to be monomorphisms of their respective kinds whose images are initial subtrees of No, and this together with the completeness of No enabled us to characterize No, up to isomorphism, as the unique complete as well as the unique nonextensible and the unique universal, s- hierarchical ordered group (s-hierarchical ordered field, etc.).

Among the striking s-hierarchical features of No that emerged from the above investigation is that much as the surreal numbers emerge from the empty set of surreal numbers by means of a transfinite recursion that provides an unfolding of the entire spectrum of numbers great and small (modulo the aforementioned provisos), the recursive process of defining No's arithmetic in turn provides an unfolding of the entire spectrum of ordered abelian number groups (ordered number fields) in such a way that an isomorphic copy of every such system either emerges as an initial subtree of No or is contained in a theoretically distinguished instance of such a system that does. In particular, it was shown that every divisible ordered abelian group (real-closed ordered field) is isomorphic to an initial subgroup (initial subfield) of No. ${ }^{1}$

Another striking s-hierarchical feature of No is that every surreal number can be assigned a canonical "proper name" (or normal form) that is a reflection of its characteristic s-hierarchical properties. These Conway names, as we call them, are expressed as formal sums of the form $\sum_{\alpha<\beta} \omega^{y_{\alpha}} \cdot r_{\alpha}$ where $\beta$ is an ordinal, $\left(y_{\alpha}\right)_{\alpha<\beta}$ is a strictly decreasing sequence of surreals, and $\left(r_{\alpha}\right)_{\alpha<\beta}$ is a sequence of nonzero real numbers, the Conway name of an ordinal being just its Cantor normal form [4, pages 31-33],[11, $\S 3.1$ and $\S 5]$.

${ }^{1}$ For subsequent developments, see [6], [13] and [14, section 7]. 
Making use of Conway names of surreal numbers, Figure 1 below offers a glimpse of the some of the early stages of the recursive unfolding of No.

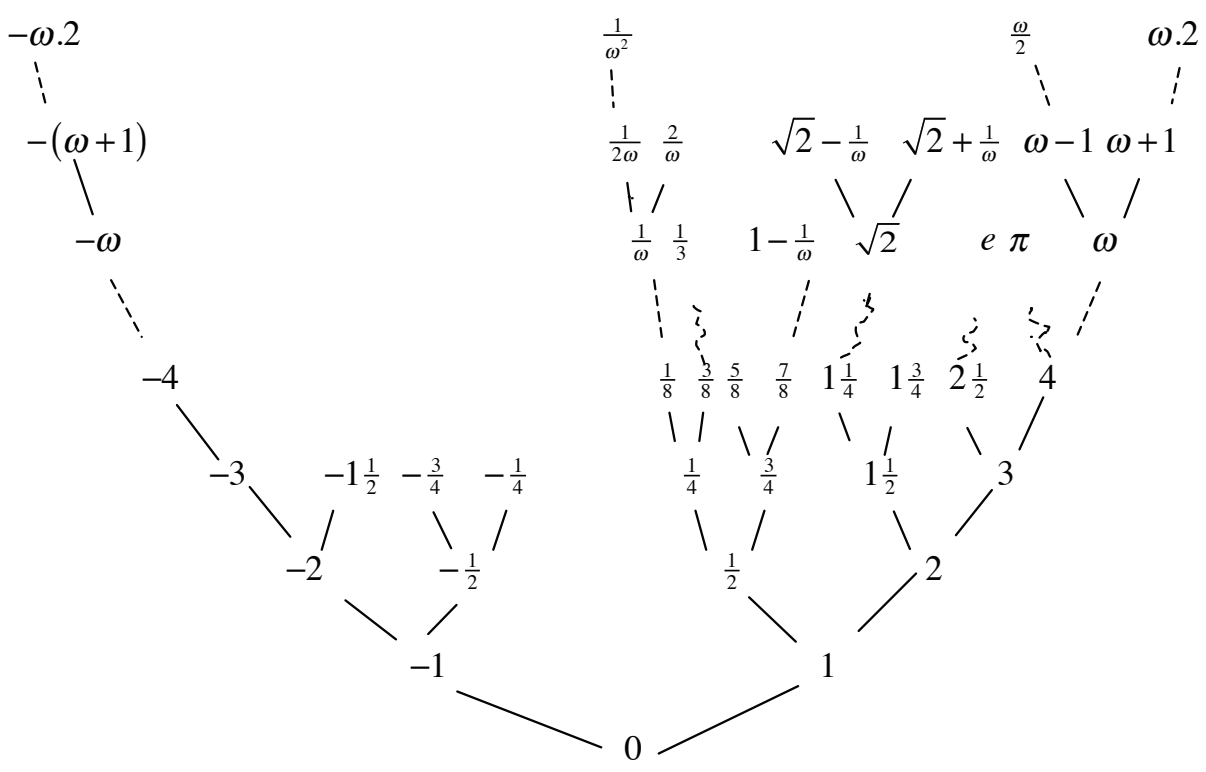

Figure 1: Early stages of the recursive unfolding of No

In the present paper we will explore some of the basic relations that exist between the Conway names of surreal numbers and the simplicity hierarchy that have heretofore been ignored. In particular, following the introduction of some preliminary material we will provide answers to the following two questions that are motivated by No's structure as a full binary tree:

(i) Given the Conway name of a surreal number, what are the Conway names of its two immediate successors?

(ii) Given the Conway names of the members of a chain of surreal numbers of limit length, what is the Conway name of the immediate successor of the chain?

Since every divisible ordered abelian group (real-closed ordered field) is isomorphic to an initial subgroup (initial subfield) of No, the answers provided to (i) and (ii) above not only shed light on the recursive unfolding of No, but on the recursive unfolding in No of divisible ordered abelian groups (real-closed ordered fields), more generally, as well as on their s-hierarchical substructures including their convex subgroups (convex subrings) and their omnific integer parts $[11, \S 5] .^{2}$ To complement the abstract treat-

\footnotetext{
${ }^{2}$ In [11] we did not mention that the omnific integer part of an s-hierarchical ordered group
} 
ment in the main body of the work, illustrations of the results that provide answers to (i) and (ii) above are collected together in Appendix A.

Throughout the paper the underlying set theory is assumed to be NBG and as such by class we mean set or proper class, the latter of which, in virtue of the axioms of global choice and foundation, always has the "cardinality" of the universe of sets. For additional information on the development of the theory of surreal numbers in NBG, we refer the reader to [8].

Acknowledgements Portions of this paper were presented at the ASL Meeting in Vienna in 2001, the Fourth Annual Algebra and Logic Colloquiumfest in Saskatoon in 2003, the Notre Dame Mathematical Logic Seminar in 2009, the Logic and Mathematics 09 conference in York, and the Joint ASL-AMS Meeting in San Francisco in 2010. We are grateful to the various organizers for affording us those opportunities, to Lou van den Dries, Mickaël Matusinski and an anonymous referee for helpful comments on improving the exposition and to Todd Eisworth for his expert assistance in creating the Latex version of the paper.

\section{Preliminaries: Lexicographically ordered binary trees}

A tree $\left\langle A,<_{s}\right\rangle$ is a partially ordered class such that for each $x \in A$, the class $\left\{y \in A: y<_{s} x\right\}$ of predecessors of $x$, written ' $p r_{A}(x)$ ', is a set well ordered by $<_{s}$. A maximal subclass of $A$ well ordered by $<_{s}$ is called a branch of the tree. Two elements $x$ and $y$ of $A$ are said to be incomparable if $x \neq y, x \nless_{s} y$ and $y \nless_{s} x$. An initial subtree of $\left\langle A,<_{s}\right\rangle$ is a subclass $A^{\prime}$ of $A$ with the induced order such that for each $x \in A^{\prime}, \operatorname{pr}_{A^{\prime}}(x)=p r_{A}(x)$. The tree-rank of $x \in A$, written ' $\rho_{A}(x)$ ', is the ordinal corresponding to the well-ordered set $\left\langle p r_{A}(x),<_{s}\right\rangle$; the $\alpha$ th level of $A$ is $\left\langle x \in A: \rho_{A}(x)=\alpha\right\rangle$; and a root of $A$ is a member of the zeroth level. If $x, y \in A$, then $y$ is said to be an immediate successor of $x$ if $x<_{s} y$ and $\rho_{A}(y)=\rho_{A}(x)+1$; and if $\left(x_{\alpha}\right)_{\alpha<\beta}$ is a chain in $A$ (i.e., a subclass of $A$ totally ordered by $<_{s}$ ), then $y$ is said to be an immediate successor of the chain if $x_{\alpha}<_{s} y$ for all $\alpha<\beta$ and $\rho_{A}(y)$ is the least ordinal greater than the tree-ranks of the members of the chain. The length of a chain $\left(x_{\alpha}\right)_{\alpha<\beta}$ in $A$ is the ordinal $\beta$.

A tree $\left\langle A,<_{s}\right\rangle$ is said to be binary if each member of $A$ has at most two immediate successors and every chain in $A$ of limit length has at most one immediate successor. If

(s-hierarchical ordered field) is itself s-hierarchical. However, to prove this it suffices to show that every predecessor of an omnific integer is itself an omnific integer, the latter of which is an immediate consequence of the second part of a theorem due to Gonshor [15, Theorem 8.1]. 
every member of $A$ has two immediate successors and every chain in $A$ of limit length (including the empty chain) has an immediate successor, then the binary tree is said to be full. Since a full binary tree has a level for each ordinal, the universe of a full binary tree is a proper class.

Using the axiom of global choice (or simply the axiom of choice, if $A$ is a set) a tree may be shown to be binary if and only if it is isomorphic to an initial subtree of the canonical full binary tree $\left\langle B,<_{B}\right\rangle$, where $B$ is the class of all sequences of $-\mathrm{s}$ and $+\mathrm{s}$ indexed over some ordinal and $x<_{B} y$ signifies that $x$ is a proper initial subsequence of $y$ [5, page 216].

As is well known, $\left\langle B,<_{B}\right\rangle$ can be totally ordered (lexicographically) in accordance with the definition: $\left(x_{\alpha}\right)_{\alpha<\mu}<_{\operatorname{lex}(B)}\left(y_{\alpha}\right)_{\alpha<\sigma}$ if and only if $x_{\beta}=y_{\beta}$ for all $\beta<$ some $\delta$, but $x_{\delta}<y_{\delta}$, it being understood that $-<$ undefined $<+$. The resulting structure $\left\langle B,<_{\operatorname{lex}(B)},<_{B}\right\rangle$ is called the lexicographically ordered canonical full binary tree.

In the theory of surreal numbers and the theory of s-hierarchical ordered systems more generally it is desirable to also have available a representation independent characterization of a lexicographically ordered binary tree. The following is one of a number of such characterizations introduced by the author in [11, page 1234].

Definition 2.1 A binary tree $\left\langle A,<_{s}\right\rangle$ together with a total ordering $<\operatorname{defined}$ on $A$ is said to be lexicographically ordered if for all distinct $x, y \in A, x$ is incomparable with $y$ if and only if $x$ and $y$ have a common predecessor lying between them.

Notational Conventions Let $\left\langle A,<_{,}\right\rangle$be a lexicographically ordered binary tree. If $(L, R)$ is a pair of subclasses of $A$ for which every member of $L$ precedes every member of $R$, then we will write ' $L<R$ '. Also, if $x$ and $y$ are members of $A$, then ' $x<_{s} y$ ' will be read " $x$ is simpler than $y$ "; and if there is an $x \in I=\{y \in A: L<\{y\}<R\}$ such that $x<_{s} y$ for all $y \in I-\{x\}$, then we will denote this simplest member of A lying between the members of $L$ and the members of $R$ by ' $\{L \mid R\}$ '. Finally, by ' $L_{s(x)}$ ' we mean $\left\{a \in A: a<_{s} x\right.$ and $\left.a<x\right\}$ and by ' $R_{s(x)}$ ' we mean $\left\{a \in A: a<_{s} x\right.$ and $\left.x<a\right\}$.

Let $\left\langle A,<_{s}\right\rangle$ be a lexicographically ordered binary tree. For each $x \in A$, there is a unique sequence $\left\{x_{\beta}: \beta<\rho_{N o}(x)\right\}$ where $x_{\beta}$ is the predecessor of $x$ in $\left\langle A,<_{s}\right\rangle$ having tree-rank $\beta$. By the sign-expansion ${ }^{3}$ of $x$, written ' $(x)$ ', we mean the sequence

\footnotetext{
${ }^{3}$ Our definition and treatment of sign-expansions differs considerably from Conway's [4, pages 29-30]. In Conway's treatment, the sign-expansions of surreal numbers (which coincide with ours) are defined using the ordered additive structure of No.
} 
$\left\{s_{\beta}(x): \beta<\rho_{N o}(x)\right\}$ defined by the condition:

$$
s_{\beta}(x)= \begin{cases}+, & \text { if } x_{\beta} \in L_{s(x)} \\ -, & \text { if } x_{\beta} \in R_{s(x)} .\end{cases}
$$

The intimate relation between lexicographically ordered binary trees and $\left\langle B,<_{\operatorname{lex}(B)},<_{B}\right\rangle$ is expressed as follows:

Proposition 2.2 (Theorem 1, [11]) Every lexicographically ordered binary tree $\left\langle A,<_{s}\right\rangle$ is isomorphic to an initial subtree of $\left\langle B,<_{\operatorname{lex}(B)},<_{B}\right\rangle$, the mapping that sends each $x \in A$ to its sign-expansion being the unique such isomorphism. If $\left\langle A,<_{s}\right\rangle$ is full, the mapping is a surjection.

The following two results collect together a number of additional properties of lexicographically ordered binary trees that will be appealed to in subsequent portions of the paper.

Proposition 2.3 (Theorem 2, [11]) Let $\left\langle A,<_{,}<_{s}\right\rangle$ be a lexicographically ordered binary tree. (i) For all $x \in A, x=\left\{L_{s(x)} \mid R_{s(x)}\right\}$; (ii) for all $x, y \in A, x<_{s} y$ if and only if $L_{s(x)}<\{y\}<R_{s(x)}$ and $y \neq x$; (iii) for all $x \in A$ and all $L, R \subseteq A, x=\{L \mid R\}$ if and only if $L$ is cofinal with $L_{s(x)}$ and $R$ is coinitial with $R_{s(x)}$.

A lexicographically ordered binary tree $\left\langle A,<,<_{s}\right\rangle$ is said to be complete [11, Definition 6], if whenever $L$ and $R$ are any two subsets of $A$ for which $L<R$, there is an $x \in A$ such that $x=\{L \mid R\}$.

Proposition 2.4 (Theorem 4, [11]) A lexicographically ordered binary tree is full if and only if it is complete.

\section{Conway names and the surreal number tree}

The new material in this section is contained in 3.3 and 3.4. The material in 3.1 and 3.2 provide the background required to keep the paper self-contained.

Journal of Logic \& Analysis 3:1 (2011) 


\subsection{Every surreal number has its own proper name: background}

With Propositions 2.2, 2.3 and 2.4 at hand, one may proceed as in [11] to develop the theory of s-hierarchical ordered structures, introduce the complete s- hierarchical ordered field $\left\langle\right.$ No $\left.,<_{,}<_{s},+,-, \cdot\right\rangle$, equate No's ordered subfield $\mathbb{R}$ of real numbers with the unique Dedekind complete initial subfield of No, and assign Conway names to the members of No making use of No's structure as a complete s-hierarchical ordered vector space over $\mathbb{R}$. In this section, we provide an overview of the salient features of the latter three steps.

To begin with, any complete lexicographically ordered binary tree can be employed to define the s-hierarchical ordered field of surreal numbers. For example, one can simply let $\left\langle\right.$ No, $\left.<_{,}<_{S}\right\rangle=\left\langle B,<_{\operatorname{lex}(B)},<_{B}\right\rangle$ (cf. [11], [15], [4, page 65]). On the other hand, this approach has the disadvantage of presupposing the existence of the ordinals and of divorcing the theory of surreal numbers from Conway's theory of games. An alternative approach due to the author [10, Appendix I], [12], [13], which uses an inductive generalization of the von Neumann ordinal construction, has neither of these drawbacks. ${ }^{4}$

With the above now said, the algebraico-tree-theoretic formulation of the central theorem in the theory of surreal numbers may be stated as follows, it being understood that $x=\left\{L_{s(x)} \mid R_{s(x)}\right\}$ and $y=\left\{L_{s(y)} \mid R_{s(y)}\right\}$.

Proposition 3.1 (Conway [4]; Ehrlich [11]) $\left\langle\mathrm{No},+, \cdot,<_{,}<_{s}\right\rangle$ is a complete $s-$ hierarchical ordered field when,+- and $\cdot$ are defined by recursion as follows where

\footnotetext{
${ }^{4}$ In these constructions, unlike Conway's [4, pages 4, 15-17], the surreal number tree is brought to the fore, making it central to the theory of surreal numbers, where the theory of shierarchical ordered structures suggests it belongs. By contrast, three chapters after introducing the surreal numbers Conway shows that the inductively defined ordered class of surreal numbers can be given the structure of a lexicographically ordered full binary tree, but this structure plays a limited role in Conway's treatment. What does play a role in Conway's treatment is a birthday function that maps each surreal number to the level of recursion at which it is created as well as the weaker notion of simplicity: $x$ is simpler than $y$, if $x$ was born prior to $y$. A surreal number's birthday corresponds to the tree rank of the surreal number in our development. Another treatment of surreal numbers in which birthdays play a crucial role was introduced by the author in [7] and subsequently employed by Alling and the author in [2] and [3]. For a discussion of how to transform the latter construction into a lexicographically ordered full binary tree, see [10, page 257]. Henceforth, however, we do not presuppose any particular definition of $\left\langle\right.$ No, $\left.<,<_{s}\right\rangle$.
} 
$x^{L}, x^{R}, y^{L}$ and $y^{R}$ are understood to range over the members of $L_{s(x)}, R_{s(x)}, L_{s(y)}$ and $R_{s(y)}$, respectively. ${ }^{5}$

Definition of $x+y$

$$
x+y=\left\{x^{L}+y, x+y^{L} \mid x^{R}+y, x+y^{R}\right\} .
$$

Definition of $-x$

$$
-x=\left\{-x^{R} \mid-x^{L}\right\}
$$

Definition of $x y$

$$
x y=\left\{x^{L} y+x y^{L}-x^{L} y^{L}, x^{R} y+x y^{R}-x^{R} y^{R} \mid x^{L} y+x y^{R}-x^{L} y^{R}, x^{R} y+x y^{L}-x^{R} y^{L}\right\} .
$$

Let $O n$ be the unique branch in $\left\langle\mathrm{No},<_{s}\right\rangle$ well ordered by $<$. The members of $O n$ are called ordinals. In this setup, each ordinal $\alpha$ emerges as the member of $O n$ of tree-rank $\alpha$ as well as the surreal number whose sign-expansion consists of $\alpha$ pluses.

Let $\mathbb{D}$ be the set of all surreal numbers having finite tree-rank and further let $\mathbb{R}=$ $\mathbb{D} \cup\{\{L \mid R\}:(L, R)$ is a Dedekind cut in $\mathbb{D}\}$.

Except for inessential changes, the following result regarding the structure of $\mathbb{R}$ is due to Conway [4, pages 12, 23-25].

Proposition 3.2 $\mathbb{R}$ (with,,$+- \cdot$ and $<$ defined à la No) is isomorphic to the ordered field of real numbers defined in any of the more familiar ways, $\mathbb{D}$ being No's ring of dyadic rationals (i.e., rationals of the form $m / 2^{n}$ where $m$ and $n$ are integers); $n=\{0, \ldots, n-1 \mid \emptyset\}$ for each positive integer $n,-n=\{\emptyset \mid-(n-1), \ldots, 0\}$ for each positive integer $n, 0=\{\emptyset \mid \emptyset\}$, and the remainder of the dyadics are the arithmetic means of their left and right predecessors of greatest tree-rank; e.g., $1 / 2=\{0 \mid 1\}$.

Two elements $a$ and $b$ of No are said to be Archimedean equivalent, if there are positive integers $m$ and $n$ such that $m|a|>|b|$ and $n|b|>|a|$; if $a$ is not Archimedean equivalent to $b$ and $|a|<|b|$, then we write ' $|a|<<|b|$ ' and $a$ is said to be infinitesimal (in absolute value) relative to $b$ and $b$ is said to be infinite (in absolute value) relative to $a$; the class of all members of No that are Archimedean equivalent to some member of No is said to constitute an Archimedean class of No. 0, which is infinitesimal (in absolute value) relative to every other surreal number, is the sole surreal number that is not a member of an Archimedean class.

\footnotetext{
${ }^{5}$ In the following definitions of,+- and $\cdot$, the set-theoretic brackets that enclose the sets of "right-sided members" and the sets of "left-sided members" are omitted (in accordance with custom).
} 
With $\mathbb{R}$ and the concept of an Archimedean class at hand, the assignment of Conway names to the members of No is carried out in three stages as follows.

One first assigns a unique appellation to the simplest positive element of each Archimedean class of No-the so-called leader of the class - by recursion as follows.

Definition 3.3 For each $y \in$ No, $\omega^{y}=\left\{0, n \omega^{L_{s(y)}} \mid \frac{1}{2^{n}} \omega^{R_{s(y)}}\right\}$ where $n$ is understood to range over all positive integers, and $\omega^{L_{s(y)}}$ and $\omega^{R_{s(y)}}$ are understood to denote typical members of $\left\{\omega^{x}: x \in L_{s(y)}\right\}$ and $\left\{\omega^{x}: x \in R_{s(y)}\right\}$, respectively.

Next, making use of No's structure as a complete s-hierarchical ordered vector space over $\mathbb{R}$, one proves the following result, whose formulation makes use of the idea of a convex subclass of an ordered class $\langle A,<\rangle$, i.e., a subclass $I$ of $A$ in which $z \in I$ whenever $x, y \in I, z \in A$ and $x<z<y$.

Proposition 3.4 (Theorem 13, [11]) For each $x \in$ No $-\{0\}$, there is a unique descending chain $I_{\alpha}^{x}, \alpha<\beta \in$ On, of convex subclasses of $\langle$ No, $<\rangle$ whose intersection contains $x$ as its simplest member, and whose components are defined by recursion as follows: $I_{\alpha}^{x}$ is the subclass of all members of No of the form $s_{\alpha}+r_{\alpha} \omega^{y_{\alpha}}+a_{\alpha}$ where (i) $s_{\alpha}=0$ if $\alpha=0$, and $s_{\alpha}$ is the simplest member of $\bigcap_{\gamma<\alpha} I_{\gamma}^{x}$, otherwise;

(ii) $r_{\alpha} \omega^{y_{\alpha}}$-henceforth called the $\alpha$-term of $x$-is the unique member of No for which $r_{\alpha} \in \mathbb{R}-\{0\}, y_{\alpha} \in$ No and $\left|x-\left(s_{\alpha}+r_{\alpha} \omega^{y_{\alpha}}\right)\right|<<\omega^{y_{\alpha}}$;

(iii) $\left|a_{\alpha}\right|<<\omega^{y_{\alpha}}$.

Since the intersection of a chain of convex subclasses is itself a convex subclass, it follows from the above result that for each $x \in \mathrm{No}-\{0\}$ there is a unique convex subclass of $\langle$ No, $<\rangle$, containing $x$ as its simplest member, consisting of all and only those surreal numbers whose $\alpha$-term is $r_{\alpha} \omega^{y_{\alpha}}$ for all $\alpha<$ some ordinal $\beta$ that depends on $x$. With this as its underlying justification, the completion of the assignment of appellations to surreal numbers is carried out as follows.

Definition 3.5 We will refer to the formal expression

$$
\sum_{\alpha<\beta} \omega^{y_{\alpha}} \cdot r_{\alpha}
$$

as the Conway name of a surreal number $x$, treat the Conway name of $x$ as a proper name of $x$ and, accordingly, write ' $x=\sum_{\alpha<\beta} \omega^{y_{\alpha}} . r_{\alpha}$ ' if and only if either $x=0$ and $\beta=0$, or $x$ is the simplest surreal number whose $\alpha$-term is $r_{\alpha} \omega^{y_{\alpha}}$ for all $\alpha<\beta$. On occasion we also write ' $\omega^{0} .0$ ' for the Conway name of 0 and at times both Conway names for zero will be employed in a given expression. 
The following result collects together three simple consequences of the above assignment of Conway names to surreal numbers that will be appealed to in subsequent portions of the paper. (i) and (ii) are immediate consequences of [11, Theorems 10-12], and (iii) is [11, Theorem 15(i)].

Proposition 3.6 Let $x, y \in$ No. Then

(i) $\omega^{x}<_{s} \omega^{y}$ if only if $x<_{s} y$;

(ii) $\omega^{x}<<\omega^{y}$ if and only if $\omega^{x}<\omega^{y}$ if and only if $x<y$;

(iii) $x<_{s} y$ whenever $x$ is a strict approximation of $y$, where $x$ is said to be a strict approximation of $y=\sum_{\alpha<\beta} \omega^{y_{\alpha}} \cdot r_{\alpha}$, if $x=\sum_{\alpha<\sigma} \omega^{y_{\alpha}} . r_{\alpha}$ for some $\sigma<\beta$.

\subsection{Sign-expansions, Conway names, and Gonshor's theorem: further background}

The two questions regarding the Conway names of surreal numbers raised in the Introduction will be answered in 3.3 and 3.4 below. Critical to our proofs of the results that collectively provide the answers to those questions are numerous applications of a theorem due to Gonshor that specifies a formula for obtaining the sign-expansion of a surreal number denoted by its Conway name from the sign-expansions of the exponents and coefficients that occur therein. Following the introduction of some notational conventions we will state Gonshor's theorem for the convenience of the reader.

Notational Conventions By ' $\overleftarrow{s}_{\tau}(y)$ ' we mean the initial subsequence of $(y)$ preceding $s_{\tau}(y)$, where, as the reader will recall, $(y)$ is the sign-expansion of $y$ and $s_{\tau}(y)$ is the $\tau$ th sign in $(y)$; in addition, following Gonshor, we will write ' $y^{+}$' for the ordinal number of pluses in the sign-expansion of $y$; and similarly, we write ' $\overleftarrow{s}_{\tau}(y)^{+}$' for the ordinal number of pluses in $\overleftarrow{s}_{\tau}(y)$

Proposition 3.7 (Gonshor's Sign-Expansion Theorem: Theorem 5.12, [15]) (1) The sign-expansion of $\omega^{y}$ where $y$ has sign-expansion $(y)$ is obtained by beginning with a

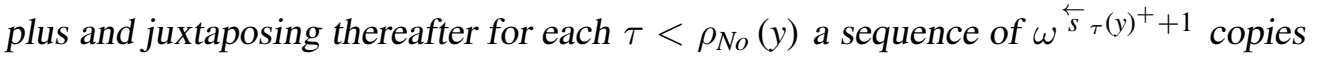
of the sign $s_{\tau}(y)$.

(2) The sign-expansion of $\omega^{y} . r$ for positive real $r$ is obtained by juxtaposing the signexpansion of $\omega^{y}$ with the sign-expansion obtained from the sign-expansion of $r$ by omitting the first plus and repeating each remaining sign $\omega^{y^{+}}$times.

(3) If $r$ is negative, the sign-expansion of $\omega^{y} . r$ is the reverse of the sign-expansion of $\omega^{y} .-r$ in the sense that every occurrence of a plus (minus) is replaced by a minus 
(plus).

(4) The sign-expansion of $\sum_{\alpha<\beta} \omega^{y_{\alpha}} . r_{\alpha}$ is obtained by juxtaposing the reduced signexpansion of $\omega^{y_{\alpha}} . r_{\alpha}$ for each of the successive $\alpha<\beta$ where the reduced signexpansion of $\omega^{y_{\alpha}} . r_{\alpha}$ is computed like the sign-expansion of $\omega^{y_{\alpha}} . r_{\alpha}$ except one employs the reduced sign-expansion of $y_{\alpha}$, the latter of which is obtained by discarding the following minuses from the sign-expansion $\left(y_{\alpha}\right)$ of $y_{\alpha}$ :

I. if $s_{\tau}\left(y_{\alpha}\right)=-$ and there is an $\nu<\alpha$ such that $s_{\delta}\left(y_{\nu}\right)=s_{\delta}\left(y_{\alpha}\right)$ for all $\delta \leq \tau$, then $s_{\tau}\left(y_{\alpha}\right)$ is discarded;

II. if $\alpha$ is a nonlimit ordinal, the sign-expansion of $y_{\alpha}$ has the sign-expansion of $y_{\alpha-1}$ followed by a minus as an initial subsequence, and $r_{\alpha-1}$ is not dyadic, then the just-mentioned minus is discarded.

In the subsequent sections of the paper we will make repeated use of the following simple consequence of Proposition 3.7(4).

Proposition 3.8 The sign-expansion of $\sum_{\alpha<\beta+1} \omega^{y_{\alpha}} . r_{\alpha}$ is equal to the sign-expansion of $\sum_{\alpha<\beta} \omega^{y_{\alpha}} . r_{\alpha}$ followed by the reduced sign-expansion of $\omega^{y_{\beta}} . r_{\beta}$.

\subsection{Conway names of immediate successors in $\left\langle\mathrm{No},<_{s}\right\rangle$}

In this section we provide a formula for obtaining the Conway names of the immediate successors of a surreal number $x$ from the Conway name of $x$. For illustrations of the formula, see Appendix A.

Notational Conventions If $x \in$ No, then by ' $x^{\mathcal{L}}$ ' we mean the immediate successor of $x$ in $\left\langle\right.$ No, $\left.<_{s}\right\rangle$ to the left (i.e. less than $x$ ) and by ' $x$ ' ' we mean the immediate successor of $x$ in $\left\langle\mathrm{No},<_{s}\right\rangle$ to the right (i.e. greater than $x$ ).

The reader will notice that the expressions $x^{\mathcal{L}}$ and $x^{\mathcal{R}}$ so defined (with calligraphic superscripts) differ in meaning from the expressions $x^{L}$ and $x^{R}$ employed in earlier sections of the paper and in the literature on surreal numbers more generally.

Conway characterized $x^{\mathcal{L}}$ and $x^{\mathcal{R}}$ for all dyadic rational numbers $x$ (see Proposition 3.2 above). Building on this, Theorems 3.11 and 3.12 below characterize $x^{\mathcal{L}}$ and $x^{\mathcal{R}}$ for all nonzero surreal numbers $x$. Moreover, if we write ' $\omega^{0} .0$ ' as the Conway name of 0 when $\beta=0$, Theorem 3.11 also holds for $x=0,0$ being the unique root of the tree [11, page 1237].

The proofs of Theorems 3.11 and 3.12 make use the following two elementary propositions that (amplify [15, Theorem 2.2a] and) refer to the simplest surreal number $\tau$ 
smaller than the members of a set of surreal numbers. The existence of $\tau$ follows from the combined facts that every nonempty convex subclass of No contains a simplest member [11, Theorem 1] and the subclass of No less than an arbitrary subset of No is a nonempty convex subclass (insofar as No contains no cofinal subset [11, page 1237]).

Proposition 3.9 Let $\tau$ be the simplest surreal number less than a surreal number $y$. Then $\tau$ is the simplest additive inverse of an ordinal less than $y$. In particular, if the sign-expansion of $y$ consists of a (possibly empty) sequence of $\alpha$ minuses, then the sign-expansion of $\tau$ consists of a sequence of $\alpha+1$ minuses, and otherwise $\tau$ is the surreal number whose sign-expansion is the longest (possibly empty) initial sequence of minuses in the sign- expansion of $y$.

Proposition 3.10 Let $\left(y_{\alpha}\right)_{\alpha<\beta}$ be a strictly decreasing sequence of surreal numbers of infinite limit length and let $\tau$ be the simplest surreal number less than all the $y_{\alpha} s$. Then $\tau$ is the simplest additive inverse of an ordinal less than all the $y_{\alpha} s$. In particular, $\tau$ is the surreal number whose sign-expansion is the shortest sequence of minuses having the (possibly empty) initial sequences of minuses in the sign-expansions of the $y_{\alpha} s$ as initial sequences (which need not be proper).

Proof Since the additive inverses of ordinals are precisely the surreal numbers whose sign-expansions consist entirely of minuses, the proofs of Propositions 3.9 and 3.10 are straightforward applications of the definition of $<_{\operatorname{lex}(B)}$ in conjunction with the fact that the mapping from surreal numbers to their sign-expansions is the unique isomorphism from $\left\langle\right.$ No,$\left.<,<_{s}\right\rangle$ onto $\left\langle B,<_{\operatorname{lex}(B)},<_{B}\right\rangle$ (see Proposition 2.2).

Theorem 3.11 Let $\sum_{\alpha<\beta+1} \omega^{y_{\alpha}} . r_{\alpha} \in$ No. Then

$$
\left(\sum_{\alpha<\beta+1} \omega^{y_{\alpha}} \cdot r_{\alpha}\right)^{\mathcal{L}}=\sum_{\alpha<\beta} \omega^{y_{\alpha}} \cdot r_{\alpha}+\left(\omega^{y_{\beta}} \cdot r_{\beta}\right)^{\mathcal{L}}
$$

and

$$
\left(\sum_{\alpha<\beta+1} \omega^{y_{\alpha}} \cdot r_{\alpha}\right)^{\mathcal{R}}=\sum_{\alpha<\beta} \omega^{y_{\alpha}} \cdot r_{\alpha}+\left(\omega^{y_{\beta}} \cdot r_{\beta}\right)^{\mathcal{R}},
$$

where $\left(\omega^{y} . r\right)^{\mathcal{L}}$ and $\left(\omega^{y} . r\right)^{\mathcal{R}}$ are given as follows for all $r \in \mathbb{R}-\{0\}$ and all $y \in$ No. (i) If $y$ is the additive inverse of an ordinal (i.e. the sign-expansion of $y$ contains no pluses) and $r$ is a dyadic rational (i.e. $r$ has an immediate successor in $\left\langle\mathbb{R},<_{s}\right\rangle$ ), then

$$
\left(\omega^{y} . r\right)^{\mathcal{L}}=\omega^{y} . r^{\mathcal{L}} \text { and }\left(\omega^{y} . r\right)^{\mathcal{R}}=\omega^{y} \cdot r^{\mathcal{R}}
$$


(ii) if $y$ is not the additive inverse of an ordinal (i.e. the sign-expansion of $y$ contains at least one plus) or $r$ is not a dyadic rational (i.e. $r$ has no immediate successor in $\left.\left\langle\mathbb{R},<_{s}\right\rangle\right)$, then

$$
\left(\omega^{y} . r\right)^{\mathcal{L}}=\omega^{y} \cdot r-\omega^{\tau} \text { and }\left(\omega^{y} . r\right)^{\mathcal{R}}=\omega^{y} \cdot r+\omega^{\tau},
$$

where $\tau$ is the simplest member of No less than $y$ (and, hence, the inverse of an ordinal).

Proof It suffices to establish that the sign-expansion of $\sum_{\alpha<\beta} \omega^{y_{\alpha}} \cdot r_{\alpha}+\left(\omega^{y_{\beta}} \cdot r_{\beta}\right)^{\mathcal{L}}$ ( $\left.\sum_{\alpha<\beta} \omega^{y_{\alpha}} \cdot r_{\alpha}+\left(\omega^{y_{\beta}} \cdot r_{\beta}\right)^{\mathcal{R}}\right)$ is the sign-expansion of $\sum_{\alpha<\beta} \omega^{y_{\alpha}} \cdot r_{\alpha}+\omega^{y_{\beta}} \cdot r_{\beta}$ followed by a minus (plus). First suppose $y_{\beta}$ is the additive inverse of an ordinal (i.e. the signexpansion of $y_{\beta}$ contains no pluses). Then the number of pluses in the reduced signexpansion of $y_{\beta}$ is zero. Accordingly, if $r_{\beta}$ is a dyadic rational, then by Proposition 3.8 the sign-expansion of $\sum_{\alpha<\beta} \omega^{y_{\alpha}} \cdot r_{\alpha}+\omega^{y_{\beta}} \cdot r_{\beta}^{\mathcal{L}}\left(\sum_{\alpha<\beta} \omega^{y_{\alpha}} \cdot r_{\alpha}+\omega^{y_{\beta}} \cdot r_{\beta}^{\mathcal{R}}\right)$ is the sign-expansion of $\sum_{\alpha<\beta} \omega^{y_{\alpha}} . r_{\alpha}$ followed by the reduced sign-expansion of $\omega^{y_{\beta}} . r_{\beta}^{\mathcal{L}}$ $\left(\omega^{y_{\beta}} \cdot r_{\beta}^{\mathcal{R}}\right.$ ), the latter (in virtue of Proposition 3.7(2, 3 and Part I of 4) being the reduced sign-expansion of $\omega^{y_{\beta}} \cdot r_{\beta}$ followed by $\omega^{y^{+}}=\omega^{0}=1$ minus (plus), thereby proving the case subsumed by (i). Now suppose $\tau$ is the simplest member of No less than $y_{\beta}$. If the sign-expansion of $y_{\beta}$ contains no pluses, by Proposition 3.9 the sign-expansion of $\tau$ consists of $\alpha+1$ minuses, where $\alpha$ is the length of the longest initial string of minuses contained in the sign-expansion of $y_{\beta}$. Thus, if in addition $r_{\beta}$ is not a dyadic rational (and therefore has no immediate successor in $\left\langle\mathbb{R},<_{s}\right\rangle$ ), then by Proposition 3.7(2,3 and Parts I and II of 4), the reduced sign-expansion of $\tau$ is the empty sequence, from which it follows that the sign-expansion of $\sum_{\alpha<\beta+1} \omega^{y_{\alpha}} \cdot r_{\alpha}-\omega^{\tau}\left(\sum_{\alpha<\beta+1} \omega^{y_{\alpha}} \cdot r_{\alpha}+\omega^{\tau}\right)$ is the sign-expansion of $\sum_{\alpha<\beta+1} \omega^{y_{\alpha}} . r_{\alpha}$ followed by a minus (plus), thereby proving one of the cases subsumed by (ii). For the remaining cases, suppose the sign-expansion of $y_{\beta}$ contains at least one plus (and, hence, that $y_{\beta} \neq 0$ ). Then $\tau=0$ if $y_{\beta}>0$, and if $y_{\beta}<0$, by Proposition 3.9 the sign-expansion of $\tau$ consists of $\alpha$ minuses, where $\alpha$ is the length of the longest initial sequence of minuses contained in the signexpansion of $y_{\beta}$. In both cases, the second in accordance with Part I of Proposition 3.7(4), the reduced sign-expansion of $\tau$ is the empty sequence, from whence it again follows that the sign-expansion of $\sum_{\alpha<\beta+1} \omega^{y_{\alpha}} \cdot r_{\alpha}-\omega^{\tau}\left(\sum_{\alpha<\beta+1} \omega^{y_{\alpha}} \cdot r_{\alpha}+\omega^{\tau}\right)$ is the sign-expansion of $\sum_{\alpha<\beta+1} \omega^{y_{\alpha}} . r_{\alpha}$ followed by a minus (plus).

Theorem 3.12 Let $\sum_{\alpha<\beta} \omega^{y_{\alpha}} . r_{\alpha} \in$ No, where $\beta$ is an infinite limit ordinal. Then

$$
\left(\sum_{\alpha<\beta} \omega^{y_{\alpha}} \cdot r_{\alpha}\right)^{\mathcal{L}}=\sum_{\alpha<\beta} \omega^{y_{\alpha}} \cdot r_{\alpha}-\omega^{\tau} \text { and }\left(\sum_{\alpha<\beta} \omega^{y_{\alpha}} \cdot r_{\alpha}\right)^{\mathcal{R}}=\sum_{\alpha<\beta} \omega^{y_{\alpha}} \cdot r_{\alpha}+\omega^{\tau},
$$


where $\tau$ is the simplest member of No less than $y_{\alpha}$ for all $\alpha<\beta$ (and, hence, the inverse of an ordinal).

Proof Let $\tau$ be the simplest member of No less than $y_{\alpha}$ for all $\alpha<\beta$. Then $\tau$ is the additive inverse of an ordinal (which may be 0); in particular, by Proposition $3.10 \tau$ is the surreal number whose sign-expansion is the shortest sequence of minuses having the initial sequences of minuses in the sign-expansions of the $y_{\alpha} \mathrm{s}$ as initial sequences. Moreover, since by Proposition 3.8 the sign- expansion of $\sum_{\alpha<\beta} \omega^{y_{\alpha}} \cdot r_{\alpha} \pm \omega^{\tau}$ is the sign-expansion of $\sum_{\alpha<\beta} \omega^{y_{\alpha}} . r_{\alpha}$ followed by the reduced sign-expansion of $\pm \omega^{\tau}$, it suffices to show that the latter consists of a single plus (minus) depending on whether \pm is a plus or a minus. But since every initial string of minuses in the sign-expansion of $\tau$ is an initial string of minuses in at least one of the $y_{\alpha} \mathrm{s}$, by Part I of Proposition 3.7(4) the reduced sign-expansion of $\pm \omega^{\tau}$ is the sign-expansion of $\pm \omega^{0}$, which consists of a single plus (minus).

\subsection{Conway names of immediate successors of chains of infinite limit length in $\left\langle\mathrm{No},<_{s}\right\rangle$}

Characterizing the Conway names of the immediate successors of chains in $\left\langle\mathrm{No},<_{s}\right\rangle$ of infinite limit length is more complicated than characterizing the Conway names of the immediate successors of individual surreal numbers. For one thing, unlike surreal numbers, chains in $\left\langle\mathrm{No},<_{s}\right\rangle$ of infinite limit length have no normal form. On the other hand, by limiting the treatment to initial chains, one can provide (with no appreciable loss of generality) a revealing taxonomy of all such chains. The absence of appreciable loss of generality accrues from the fact that every chain in $\left\langle\mathrm{No},<_{s}\right\rangle$ of infinite limit length is a cofinal subchain of an initial chain in $\left\langle\mathrm{No},{ }_{s}\right\rangle$ whose immediate successors coincide.

Notational Convention If $x=\left(x_{\alpha}\right)_{\alpha<\mu}$ is a chain in $\left\langle\right.$ No, $\left.<_{s}\right\rangle$ of infinite limit length, then by ' $\sigma(x)$ ' we mean the immediate successor of the chain.

The taxonomy of initial chains of surreal numbers of infinite limit length alluded to above is encapsulated by the following result.

Theorem 3.13 Let $x$ be an initial chain in $\left\langle\mathrm{No},<_{s}\right\rangle$ of infinite limit length. Then $x$ contains a cofinal subchain of one of the following forms.

(I) $x$ contains a cofinal subchain (of infinite limit length) of the form

$$
\left(\sum_{\alpha<\sigma} \omega^{y_{\alpha}} \cdot r_{\alpha}\right)_{\sigma<\beta} .
$$

Journal of Logic \& Analysis 3:1 (2011) 
(II) $x$ contains a cofinal subchain (of infinite limit length) of the form

$$
\left(\sum_{\alpha<\beta} \omega^{y_{\alpha}} \cdot r_{\alpha} \pm \omega^{z_{\mu}}\right)_{\mu<\nu},
$$

where $\left(\omega^{z \mu}\right)_{\mu<\nu}$ is a chain of leaders (of infinite limit length) such that $\omega^{y_{\alpha}}>\omega^{z_{\mu}}$ for all $\alpha<\beta$ and all $\mu<\nu$.

(III) $x$ contains a cofinal subchain (of infinite limit length) of the form

$$
\left(\sum_{\alpha<\beta} \omega^{y_{\alpha}} \cdot r_{\alpha}+\omega^{y_{\beta}} \cdot a_{n}\right)_{n<\omega},
$$

where $\beta \geq 0$ and $\left(a_{n}\right)_{n<\omega}$ is an initial chain in $\left\langle\mathbb{R},<_{s}\right\rangle$ having one of the following forms:

(i) $\left(a_{n}\right)_{n<\omega}$ has an immediate successor in $\left\langle\mathbb{R},<_{s}\right\rangle$;

(ii) there is an $i \geq 0$ such that $a_{i}<a_{i+1}$ and $a_{i+1}>a_{i+2}>\cdots>a_{i+n}>\ldots$;

(iii) there is an $i \geq 0$ such that $a_{i}>a_{i+1}$ and $a_{i+1}<a_{i+2}<\cdots<a_{i+n}<\ldots$;

(iv) $a_{n}=n$ or $a_{n}=-n$ (henceforth $a_{n}= \pm n$ ) for all $n \geq 0$.

Proof Let $x$ be an initial chain in $\left\langle\right.$ No, $\left.<_{s}\right\rangle$ of infinite limit length. Since (by Proposition 3.6(iii)) every strict approximation of a surreal number is simpler than the given surreal number, it follows that there is a subchain of $x$, say, $x^{\prime}$, consisting (in increasing length) of all the strict approximations of $\sigma(x)$. If $x^{\prime}$ is cofinal in $x$, then (I) is the case. If $x^{\prime}$ is not cofinal in $x$, there is a longest strict approximation of $\sigma(x)$ in $x$, say, $\sum_{\alpha<\beta} \omega^{y_{\alpha}} \cdot r_{\alpha}$ (which may be 0 , i.e., $\beta=0$ ). But then, in virtue of Proposition 3.7(1 and 4) and the fact that $\omega^{a}<_{s} \omega^{b}$ whenever $a<_{s} b$ and, hence, whenever the sign-expansion of $a$ is an initial sequence of the sign-expansion of $b$, there is a maximal subchain $x^{\prime \prime}$ of $x$ of the form

$$
\left(\sum_{\alpha<\beta} \omega^{y_{\alpha}} \cdot r_{\alpha} \pm \omega^{z \mu}\right)_{\mu<\nu}
$$

where $\left(\omega^{z \mu}\right)_{\mu<\nu}$ is a chain of leaders in $\left\langle\right.$ No, $\left.<_{s}\right\rangle$ such that $\omega^{y_{\alpha}}>\omega^{z_{\mu}}$ for all $\alpha<\beta$ and all $\mu<\nu$. (Note if $\beta=0$, the condition $\omega^{y_{\alpha}}>\omega^{z_{\mu}}$ for all $\alpha<\beta$ and all $\mu<\nu$ holds vacuously.) If $x^{\prime \prime}$ is cofinal with $x$, then the chain of leaders is of infinite limit length and (II) is the case. However, if $x^{\prime \prime}$ is not cofinal with $x$, it follows from Proposition 3.7(1 and 4) that there is a last (i.e. least simple) member of $x^{\prime \prime}$, which may be written

$$
\sum_{\alpha<\beta} \omega^{y_{\alpha}} \cdot r_{\alpha} \pm \omega^{y_{\beta}}
$$


By appealing to Proposition 3.7(2-4), it is not difficult to see that there must be a cofinal subchain of $x$ of the form

$$
\left(\sum_{\alpha<\beta} \omega^{y_{\alpha}} \cdot r_{\alpha}+\omega^{y_{\beta}} \cdot a_{n}\right)_{n<\omega}
$$

where $\left(a_{n}\right)_{n<\omega}$ is an initial chain in $\left\langle\mathbb{R},<_{s}\right\rangle$. Notice that the chain of real numbers must be infinite, otherwise $\sum_{\alpha<\beta} \omega^{y_{\alpha}} . r_{\alpha}$ would not be the maximal approximation of $\sigma(x)$ in $x$, contrary to assumption. To complete the proof it suffices to note that there are five possible cases corresponding to the above listed five mutually exclusive, collectively exhaustive types of maximal chains there are in $\left\langle\mathbb{R},<_{s}\right\rangle$, (iv) constituting two cases.

As we mentioned above, Theorem 3.13 provides a taxonomy of the types of initial chains in $\left\langle\mathrm{No},<_{s}\right\rangle$ of infinite limit length. In the remainder of this section we establish four theorems that collectively characterize the immediate successors of those chains. As the last three of these theorems make clear, the initial taxonomy is rough since some of the cases have multiple subcases. Illustrations of the cases and subcases are contained in Appendix A.

Theorem 3.14 Let $x$ be an initial chain in $\left\langle\mathrm{No},<_{s}\right\rangle$ of infinite limit length. If $x$ contains a cofinal subchain of the form $\left(\sum_{\alpha<\sigma} \omega^{y_{\alpha}} \cdot r_{\alpha}\right)_{\sigma<\beta}$, then $\sigma(x)=\sum_{\alpha<\beta} \omega^{y_{\alpha}} \cdot r_{\alpha}$.

Proof Since (by Proposition 3.6(iii)) every strict approximation of a surreal number is simpler than the given surreal number, and since (by Definition 3.5) $\sum_{\alpha<\beta} \omega^{y_{\alpha}} \cdot r_{\alpha}$ is the simplest surreal number whose $\alpha$-term is $r_{\alpha} \omega^{y_{\alpha}}$ for all $\alpha<\beta$, the theorem follows.

Theorem 3.15 Let $x$ be an initial chain in $\left\langle\mathrm{No},{ }_{s}\right\rangle$ of infinite limit length. If $x$ contains a cofinal subchain of the form $\left(\sum_{\alpha<\beta} \omega^{y_{\alpha}} . r_{\alpha} \pm \omega^{z \mu}\right)_{\mu<\nu}$, where $\left(\omega^{z \mu}\right)_{\mu<\nu}$ is a chain of leaders (of infinite limit length) such that $\omega^{y_{\alpha}}>\omega^{z_{\mu}}$ for all $\alpha<\beta$ and all $\mu<\nu$, then one of the following is the case.

(i) Either $\beta$ is a limit ordinal (which may be 0 ) or $\beta$ is a successor ordinal and $\omega^{y_{\beta-1}}$ is not the immediate successor of the chain $\left(\omega^{z_{\mu}}\right)_{\mu<\nu}$, in which case

$$
\sigma(x)=\sum_{\alpha<\beta} \omega^{y_{\alpha}} \cdot r_{\alpha} \pm \omega^{\sigma\left(\left(z_{\mu}\right)_{\mu<\nu}\right)} ;
$$


(ii) $\beta$ is a successor ordinal, $r_{\beta-1}$ is a nonzero dyadic rational and $\omega^{y_{\beta-1}}$ is the immediate successor of the chain $\left(\omega^{z \mu}\right)_{\mu<\nu}$, in which case

$$
\sigma(x)=\sum_{\alpha<\beta-1} \omega^{y_{\alpha}} \cdot r_{\alpha}+\omega^{y_{\beta-1}} \cdot r_{\beta-1}^{\mathcal{R}} \text { or } \sigma(x)=\sum_{\alpha<\beta-1} \omega^{y_{\alpha}} \cdot r_{\alpha}+\omega^{y_{\beta-1}} \cdot r_{\beta-1}^{\mathcal{L}}
$$

depending on whether \pm is + or - , respectively;

(iii) $\beta$ is a successor ordinal, $r_{\beta-1}$ is not a dyadic rational and $\omega^{y_{\beta-1}}$ is the immediate successor of the chain $\left(\omega^{z_{\mu}}\right)_{\mu<\nu}$, in which case

$$
\sigma(x)=\sum_{\alpha<\beta-1} \omega^{y_{\alpha}} \cdot r_{\alpha}+\omega^{y_{\beta-1}} \cdot r_{\beta-1} \pm \omega^{y_{\beta}^{\mathcal{L}}} .
$$

Proof Since $\left(z_{\mu}\right)_{\mu<\nu}$ is a chain of infinite limit length where $y_{\alpha}>z_{\mu}$ for all $\alpha<\beta$ and all $\mu<\nu, y_{\alpha}>\sigma\left(\left(z_{\mu}\right)_{\mu<\nu}\right)$ for all $\alpha<\beta$ if $\beta$ is a limit ordinal, and $y_{\beta-1} \geq \sigma\left(\left(z_{\mu}\right)_{\mu<\nu}\right)$ if $\beta$ is a successor ordinal. Thus, if either hypothesis in (i) is the case, then $y_{\alpha}>\sigma\left(\left(z_{\mu}\right)_{\mu<\nu}\right)$ for all $\alpha<\beta$, and so $\omega^{y_{\alpha}}>\omega^{\sigma\left(\left(z_{\mu}\right)_{\mu<\nu}\right)}$ for all $\alpha<\beta$. In these cases the sign-expansion that results by taking the union of the signexpansions of the members of the specified cofinal subchain is the juxtaposition of the sign-expansion of $\sum_{\alpha<\beta} \omega^{y_{\alpha}} . r_{\alpha}$ followed by the union of the reduced sign-expansions of the $\omega^{z_{\mu}} \mathrm{s}$. But this is the juxtaposition of the sign-expansion of $\sum_{\alpha<\beta} \omega^{y_{\alpha}} . r_{\alpha}$ followed by the reduced sign-expansion of $\pm \omega^{\sigma\left(\left(z_{\mu}\right)_{\mu<\nu}\right)}$, which proves (i). Now suppose $\beta$ is a successor ordinal. If $\omega^{y_{\beta-1}}$ is the immediate successor of the chain $\left(\omega^{z \mu}\right)_{\mu<\nu}$, then since each of the $z_{\mu} \mathrm{s}$ is an initial subsequence of $y_{\beta-1}$, all of the minuses in the sign-expansions of the $z_{\mu}$ s are deleted when computing the reduced sign-expansions of the $\omega^{z \mu} \mathrm{s}$. Accordingly, in this case the sign-expansion that results by taking the union of the sign- expansions of the members of the cofinal subchain is the juxtaposition of the sign-expansion of $\sum_{\alpha<\beta-1} \omega^{y_{\alpha}} \cdot r_{\alpha}+\omega^{y_{\beta-1}} \cdot r_{\beta-1}$ followed by $\omega^{\left(y_{\beta-1}\right)^{+}}$ pluses (minuses) depending on whether \pm is + or - , respectively. But in virtue of Proposition 3.7(4), this is the sign-expansion of $\sum_{\alpha<\beta-1} \omega^{y_{\alpha}} \cdot r_{\alpha}+\omega^{y_{\beta-1}} \cdot r_{\beta-1}^{\mathcal{R}}$ $\left(\sum_{\alpha<\beta-1} \omega^{y_{\alpha}} \cdot r_{\alpha}+\omega^{y_{\beta-1}} \cdot r_{\beta-1}^{\mathcal{L}}\right)$ if $r_{\beta-1}$ is a nonzero dyadic rational, and the signexpansion of $\sum_{\alpha<\beta-1} \omega^{y_{\alpha}} \cdot r_{\alpha}+\omega^{y_{\beta-1}} \cdot r_{\beta-1} \pm \omega^{y_{\beta}^{\mathcal{L}}}$ if $r_{\beta-1}$ is not a dyadic rational. The proof of (ii), the dyadic case, uses Part I of Proposition 3.7(4) and the proof of (iii), the nondyadic case, uses Parts I and II of Proposition 3.7(4), the latter of which requires that the final minus in $y_{\beta-1}^{\mathcal{L}}$ be deleted when computing the reduced sign-expansion of $y_{\beta-1}^{\mathcal{L}}$. 
Theorem 3.16 Let $x$ be an initial chain in $\left\langle\mathrm{No},<_{s}\right\rangle$ of infinite limit length that contains a cofinal subchain of the form

$$
\left(\sum_{\alpha<\beta} \omega^{y_{\alpha}} \cdot r_{\alpha}+\omega^{y_{\beta}} \cdot a_{n}\right)_{n<\omega}
$$

where $\left(a_{n}\right)_{n<\omega}$ is an initial chain in $\left\langle\mathbb{R},<_{s}\right\rangle$.

(i) If $\left(a_{n}\right)_{n<\omega}$ has an immediate successor in $\left\langle\mathbb{R},<_{s}\right\rangle$, say, $r_{\beta}$, then

$$
\sigma(x)=\sum_{\alpha<\beta} \omega^{y_{\alpha}} \cdot r_{\alpha}+\omega^{y_{\beta}} \cdot r_{\beta}
$$

(ii) if there is an $i \geq 0$ such that $a_{i}<a_{i+1}$ and $a_{i+1}>a_{i+2}>\cdots>a_{i+n}>\ldots$, then

$$
\begin{aligned}
\sigma(x) & =\sum_{\alpha<\beta} \omega^{y_{\alpha}} \cdot r_{\alpha}+\omega^{y_{\beta}} \cdot a_{i}+\omega^{y_{\beta}^{\mathcal{L}}} \\
& \left.=\sum_{\alpha<\beta} \omega^{y_{\alpha}} \cdot r_{\alpha}+\omega^{y_{\beta}^{\mathcal{L}}}, \text { if } i \text { (and, hence, } a_{i}\right)=0 ;
\end{aligned}
$$

(iii) if there is an $i \geq 0$ such that $a_{i}>a_{i+1}$ and $a_{i+1}<a_{i+2}<\cdots<a_{i+n}<\ldots$, then

$$
\begin{aligned}
\sigma(x) & =\sum_{\alpha<\beta} \omega^{y_{\alpha}} \cdot r_{\alpha}+\omega^{y_{\beta}} \cdot a_{i}-\omega^{y_{\beta}^{\mathcal{L}}} \\
& \left.=\sum_{\alpha<\beta} \omega^{y_{\alpha}} \cdot r_{\alpha}-\omega^{y_{\beta}^{\mathcal{L}}}, \text { if } i \text { (and, hence, } a_{i}\right)=0 .
\end{aligned}
$$

Proof If (i) is the case, then the chain $\left(a_{n}\right)_{n<\omega}$ has an immediate successor in $\left\langle\mathbb{R},<_{s}\right\rangle$, say, $r_{\beta}$. And so, by Proposition 3.7(2-4),

$$
\sigma(x)=\sum_{\alpha<\beta} \omega^{y_{\alpha}} \cdot r_{\alpha}+\omega^{y_{\beta}} \cdot r_{\beta} .
$$

If (ii) is the case, then there is an $i \geq 0$ such that $a_{i}\left\langle a_{i+1}\right.$ and $\left.a_{i+1}>a_{i+2}>\cdots\right\rangle$ $a_{i+n}>\ldots$. Notice that insofar as the sign-expansion of each member of the sequence

$$
\left(\sum_{\alpha<\beta} \omega^{y_{\alpha}} \cdot r_{\alpha}+\omega^{y_{\beta}} \cdot a_{i+n}\right)_{n<\omega}
$$

is an initial subsequence of the sign-expansions of the subsequent members of the sequence, the sign-expansion that results by taking the union of the sign-expansions of the members of the sequence is the sign-expansion of $\sum_{\alpha<\beta} \omega^{y_{\alpha}} \cdot r_{\alpha}+\omega^{y_{\beta}} \cdot a_{i}$ followed by $\omega^{y_{\beta}^{+}}$pluses, the latter being the contribution from $a_{i+1}=a_{i}^{\mathcal{R}}$, followed by a sequence of $\omega^{y_{\beta}^{+}}$minuses for each $a_{i+n}$ where $n>1$; that is, the sign- expansion 
of $\sum_{\alpha<\beta} \omega^{y_{\alpha}} \cdot r_{\alpha}+\omega^{y_{\beta}} \cdot a_{i}$ followed by $\omega^{y_{\beta}^{+}}$pluses followed by $\omega^{y_{\beta}^{+}} \cdot \omega^{1}=\omega^{y_{\beta}^{+}+1}$ minuses. Accordingly, to complete the proof for (ii), it suffices to note that, (a) the sign-expansion of

$$
\sum_{\alpha<\beta} \omega^{y_{\alpha}} \cdot r_{\alpha}+\omega^{y_{\beta}} \cdot a_{i}+\omega^{y_{\beta}^{\mathcal{L}}}
$$

is equal to the sign-expansion of $\sum_{\alpha<\beta} \omega^{y_{\alpha}} \cdot r_{\alpha}+\omega^{y_{\beta}} \cdot a_{i}$ followed by the reduced sign-expansion of $\omega^{y_{\beta}^{\mathcal{L}}}$ and that, (b) the reduced sign-expansion of $\omega^{y_{\beta}^{\mathcal{L}}}$ is $\omega^{y_{\beta}^{+}}$pluses followed by $\omega^{y_{\beta}^{+}+1}$ minuses, the latter being the contribution from the single minus occurring in the reduced sign-expansion of $y_{\beta}^{\mathcal{L}}$, the minuses, if any, from the signexpansion of $y_{\beta}$ having been discarded in accordance with Part I of Proposition 3.7(4). Notice that the just-mentioned minus must indeed occur in the reduced sign-expansion of $y_{\beta}^{\mathcal{L}}$, for otherwise the sign-expansion of $y_{\beta}^{\mathcal{L}}$ would be an initial sequence of the sign-expansion of some $y_{\alpha}$ where $\alpha<\beta$, thereby rendering the just-said $y_{\alpha}<y_{\beta}$, contrary to assumption.

The proof for (iii) is the same as for (ii) except that here we have the sign-expansion of $\sum_{\alpha<\beta} \omega^{y_{\alpha}} . r_{\alpha}+\omega^{y_{\beta}} . a_{i}$ followed by $\omega^{y_{\beta}^{+}}$minuses, the latter being the contribution from $a_{i+1}=a_{i}^{\mathcal{L}}$, followed by $\omega^{y_{\beta}^{+}}$pluses for each $a_{i+n}$ where $n>1$; and in the second part of the proof we use the fact that the reduced sign- expansion of $-\omega^{y_{\beta}^{\mathcal{L}}}$ results from replacing the pluses and minuses in the reduced sign-expansion of $\omega^{y_{\beta}^{\mathcal{L}}}$ with minuses and pluses, respectively, in accordance with Proposition 3.7(3).

Our final theorem characterizing immediate successors of chains of infinite limit length addresses the chains described in part III(iv) of 3.13. There are five special cases. Our proof of the last case makes use of the following simple result.

Proposition 3.17 For all $x, y \in$ No, if $y<x<y^{\mathcal{R}}$, then $y \in L_{s(x)}$.

Proof Suppose $y<x<y^{\mathcal{R}}$. Since $L_{s\left(y^{\mathcal{R}}\right)}=L_{s(y)} \cup\{y\}$ and $\left\{y^{\mathcal{R}}\right\}<R_{s\left(y^{\mathcal{R}}\right)}$, it is evident that $L_{s\left(y^{\mathcal{R}}\right)}<\{x\}<R_{s(y \mathcal{R})}$. But then, by Proposition 2.3(ii) and the fact that $y^{\mathcal{R}} \neq x$, we have $y^{\mathcal{R}}<_{s} x$ and, hence, that $y<_{s} x$ since $y<_{s} y^{\mathcal{R}}$. Accordingly, $y \in L_{s(x)}$, insofar as $y<x$.

Theorem 3.18 Let $x$ be an initial chain in $\left\langle\mathrm{No},<_{s}\right\rangle$ of infinite limit length that contains a cofinal subchain of the form

$$
\left(\sum_{\alpha<\beta} \omega^{y_{\alpha}} \cdot r_{\alpha} \pm \omega^{y_{\beta}} \cdot n\right)_{n<\omega} .
$$


(i) If $y_{\alpha}>y_{\beta}^{\mathcal{R}}$ for all $\alpha<\beta$, then

$$
\sigma(x)=\sum_{\alpha<\beta} \omega^{y_{\alpha}} \cdot r_{\alpha} \pm \omega^{y_{\beta}^{\mathcal{R}}}
$$

(ii) if $\beta$ is a successor ordinal, $y_{\beta-1} \leq y_{\beta}^{\mathcal{R}}$, there is no $z \in L_{s\left(y_{\beta-1}\right)}$ such that $\rho_{N o}(z)>\rho_{N o}\left(y_{\beta}\right)$, and $r_{\beta-1}$ is a nonzero dyadic rational, then

$$
\sigma(x)=\sum_{\alpha<\beta-1} \omega^{y_{\alpha}} \cdot r_{\alpha}+\omega^{y_{\beta-1}} \cdot r_{\beta-1}^{\mathcal{R}} \text { or } \sigma(x)=\sum_{\alpha<\beta-1} \omega^{y_{\alpha}} \cdot r_{\alpha}+\omega^{y_{\beta-1}} \cdot r_{\beta-1}^{\mathcal{L}}
$$

depending on whether \pm is + or - , respectively;

(iii) if $\beta$ is a successor ordinal, $y_{\beta-1} \leq y_{\beta}^{\mathcal{R}}$, there is no $z \in L_{s\left(y_{\beta-1}\right)}$ such that $\rho_{N o}(z)>\rho_{N o}\left(y_{\beta}\right)$, and $r_{\beta-1}$ is not a dyadic rational, then

$$
\sigma(x)=\sum_{\alpha<\beta} \omega^{y_{\alpha}} \cdot r_{\alpha} \pm \omega^{y_{\beta}^{\mathcal{L}}}
$$

(iv) if $\beta$ is a successor ordinal, $y_{\beta-1} \leq y_{\beta}^{\mathcal{R}}$, and there is a $z \in L_{s\left(y_{\beta-1}\right)}$ such that $\rho_{\text {No }}(z)>\rho_{N o}\left(y_{\beta}\right)$, then

$$
\begin{aligned}
\sigma(x) & =\sum_{\alpha<\beta} \omega^{y_{\alpha}} \cdot r_{\alpha} \pm \omega^{\left\{y_{\beta} \mid y_{\beta-1}\right\}} \\
& =\sum_{\alpha<\beta} \omega^{y_{\alpha}} \cdot r_{\alpha} \pm \omega^{y}
\end{aligned}
$$

where $y$ is the simplest $z \in L_{s\left(y_{\beta-1}\right)}$ such that $\rho_{N o}(z)>\rho_{N o}\left(y_{\beta}\right)$;

(v) if $\beta$ is an infinite limit ordinal and $y_{\nu}<y_{\beta}^{\mathcal{R}}$ for some $\nu<\beta$, then

$$
\sigma(x)=\sum_{\alpha<\beta} \omega^{y_{\alpha}} \cdot r_{\alpha} \pm \omega\left\{y_{\beta} \mid\left(y_{\alpha}\right)_{\alpha<\beta}\right\} .
$$

Proof Suppose $x$ has the cofinal subchain of the form

$$
\left(\sum_{\alpha<\beta} \omega^{y_{\alpha}} \cdot r_{\alpha} \pm \omega^{y_{\beta}} \cdot n\right)_{n<\omega} .
$$

Then the sign-expansion that results by taking the union of the sign-expansions of the members of the sequence is the sign-expansion of $\sum_{\alpha<\beta} \omega^{y_{\alpha}} \cdot r_{\alpha}$ followed by the reduced sign-expansion of $\omega^{y_{\beta}}\left(-\omega^{y_{\beta}}\right)$ followed by $\omega^{y_{\beta}^{+}} \cdot \omega^{1}=\omega^{y^{+}+1}$ pluses (minuses), the latter signs coming from the $\omega^{y_{\beta}^{+}}$pluses (minuses) added for each of the $\omega^{1}$ new pluses (minuses) that are contributed by the successive coefficients $n(-n)$ for 
$n>1$. There are five mutually exclusive and collectively exhaustive cases to consider specified in (i)-(v) above. We consider these in turn.

Suppose $y_{\alpha}>y_{\beta}^{\mathcal{R}}$ for all $\alpha<\beta$. Since the sign-expansion of $y_{\beta}^{\mathcal{R}}$ is the sign-expansion of $y_{\beta}$ followed by a plus, and since $y_{\alpha}>y_{\beta}^{\mathcal{R}}$ for all $\alpha<\beta$, the sign- expansion of $\sum_{\alpha<\beta} \omega^{y_{\alpha}} \cdot r_{\alpha} \pm \omega^{y_{\beta}^{\mathcal{R}}}$ is the sign-expansion of $\sum_{\alpha<\beta} \omega^{y_{\alpha}} \cdot r_{\alpha}$ followed by the reduced sign-expansion of $\omega^{y_{\beta}^{\mathcal{R}}}$, where the reduced sign-expansion of $y_{\beta}^{\mathcal{R}}$ is the reduced signexpansion of $y_{\beta}$ followed by a plus. But then, in virtue of Proposition 3.7(1,2 and 4), the sign-expansion of $\sum_{\alpha<\beta} \omega^{y_{\alpha}} . r_{\alpha} \pm \omega^{y_{\beta}^{\mathcal{R}}}$ is the sign- expansion of $\sum_{\alpha<\beta} \omega^{y_{\alpha}} . r_{\alpha}$ followed by the reduced sign-expansion of $\omega^{y_{\beta}}\left(-\omega^{y_{\beta}}\right)$ followed by $\omega^{y^{+}+1}$ pluses (minuses), which proves (i). Next suppose $\beta$ is not a limit ordinal and $y_{\beta-1} \leq y_{\beta}^{\mathcal{R}}$. Since $y_{\beta-1} \leq y_{\beta}^{\mathcal{R}}$, it follows from Proposition 3.17 that $y_{\beta} \in L_{s\left(y_{\beta-1}\right)}$ and, hence, that the sign-expansion of $y_{\beta}$ is an initial subsequence of the sign-expansion of $y_{\beta-1}$, from which it follows that there are no minuses in the reduced sign-expansion of $y_{\beta}$. Accordingly, in this case the sign-expansion that results by taking the union of the signexpansions of the members of the sequence is the juxtaposition of the sign-expansion of $\sum_{\alpha<\beta-1} \omega^{y_{\alpha}} \cdot r_{\alpha}+\omega^{y_{\beta-1}} . r_{\beta-1}$ followed by $\omega^{y_{\beta}^{+}}$pluses (minuses) followed by $\omega^{y_{\beta}^{+}}$. $\omega^{1}=\omega^{y_{\beta}^{+}+1}$ pluses (minuses) depending on whether \pm is + or - , respectively. But since the cardinality of $\omega^{y_{\beta}^{+}+1}$ is infinitely greater than the cardinality of $\omega^{y_{\beta}^{+}}$, this results in the juxtaposition of the sign-expansion of $\sum_{\alpha<\beta-1} \omega^{y_{\alpha}} \cdot r_{\alpha}+\omega^{y_{\beta-1}} \cdot r_{\beta-1}$ followed by $\omega^{y_{\beta}^{+}+1}$ pluses (minuses). There are two cases to consider.

First suppose there is no $z \in L_{s\left(y_{\beta-1}\right)}$ such that $\rho_{N o}(z)>\rho_{N o}\left(y_{\beta}\right)$. Then the signexpansion of $y_{\beta-1}$ is the sign-expansion of $y_{\beta}$ followed by a single plus followed by a string of minuses, the string of minuses being empty when $y_{\beta-1}=y_{\beta}^{\mathcal{R}}$. But, in virtue of Proposition 3.7(4), this is the sign-expansion of $\sum_{\alpha<\beta-1} \omega^{y_{\alpha}} \cdot r_{\alpha}+\omega^{y_{\beta-1}} \cdot r_{\beta-1}^{\mathcal{R}}$ $\left(\sum_{\alpha<\beta-1} \omega^{y_{\alpha}} \cdot r_{\alpha}+\omega^{y_{\beta-1}} \cdot r_{\beta-1}^{\mathcal{L}}\right)$ if $r_{\beta-1}$ is a dyadic rational, and the sign-expansion of $\sum_{\alpha<\beta-1} \omega^{y_{\alpha}} \cdot r_{\alpha}+\omega^{y_{\beta-1}} \cdot r_{\beta-1} \pm \omega^{y_{\beta-1}^{\mathcal{L}}}$ if $r_{\beta-1}$ is not a dyadic rational. The proof of the dyadic case uses Part I of Proposition 3.7(4) and the proof of the nondyadic case uses Parts I and II of Proposition 3.7(4), which collectively imply that the reduced sign-expansion of $y_{\beta-1}$ is equal to the reduced sign-expansion of $y_{\beta-1}^{\mathcal{L}}$, when $r_{\beta-1}$ is not a dyadic rational, and hence that the contribution from the reduced sign- expansion of $y_{\beta-1}^{\mathcal{L}}$ is $\omega^{y_{\beta}^{+}+1}$ pluses (minuses) depending on whether \pm is + or - , thereby proving (ii) and (iii).

Turning now to (iv), suppose there is a $z \in L_{s\left(y_{\beta-1}\right)}$ such that $\rho_{N o}(z)>\rho_{N o}\left(y_{\beta}\right)$, and let $y$ be the simplest such $z$. Then the sign-expansion of $y_{\beta-1}$ has an initial sequence 
consisting of the sign-expansion of $y_{\beta}$ followed by a single plus followed by a nonempty string of minuses followed by a plus, the subsequence up to but not including the plus being the sign-expansion of $y$. Since the sign-expansion of $y_{\beta}$ is an initial sequence of the sign- expansion of $y$ which in turn is an initial sequence of the sign-expansion of $y_{\beta-1}$, the reduced sign-expansion of $y$ obtained in the computation of the sign-expansion of $\sum_{\alpha<\beta-1} \omega^{y_{\alpha}} \cdot r_{\alpha}+\omega^{y_{\beta-1}} \cdot r_{\beta-1} \pm \omega^{y}$ consists of all the pluses in $y_{\beta}$ followed by a single plus. But then, the sign-expansion of $\sum_{\alpha<\beta-1} \omega^{y_{\alpha}} \cdot r_{\alpha}+\omega^{y_{\beta-1}} \cdot r_{\beta-1} \pm \omega^{y}$ is the sign-expansion of $\sum_{\alpha<\beta-1} \omega^{y_{\alpha}} \cdot r_{\alpha}+\omega^{y_{\beta-1}} \cdot r_{\beta-1}$ followed by $\omega^{y_{\beta}^{+}+1}$ pluses (minuses) depending on whether \pm is + or - , thereby completing the proof of (iv).

Finally, suppose $\beta$ is a limit ordinal and $y_{\nu}<y_{\beta}^{\mathcal{R}}$ for some $\nu<\beta$. Then there is a least $\tau<\beta$ such that $y_{\alpha}<y_{\beta}^{\mathcal{R}}$ for all $\tau \leq \alpha<\beta$. Moreover, in virtue of Proposition 3.17, $y_{\beta} \in L_{s\left(y_{\alpha}\right)}$ for all such $\alpha$. It is not difficult to see that for all $\alpha$ such that $\tau \leq \alpha<\beta$ the sign-expansion of $y_{\alpha}$ has an initial subsequence consisting of the sign-expansion of $y_{\beta}$ followed by a single plus followed by a maximal string of minuses, i.e. a string of minuses that is either followed by a plus or no signs at all. Let $\left(x_{\alpha}\right)_{\alpha<\rho}$ be the chain of surreal numbers having the just-described chain of sign-expansions that end in maximal strings of minuses, and let $y$ be the surreal number whose sign-expansion is the union of the chain of sign-expansions of the $x_{\alpha}$ s. If $\rho$ is a successor ordinal, $y=x_{\rho-1}$, and if $\rho$ is an infinite limit ordinal, $y=\sigma\left(\left(x_{\alpha}\right)_{\alpha<\rho}\right)$; and in both cases it is easy to see that $y=\left\{y_{\beta} \mid\left(y_{\alpha}\right)_{\alpha<\beta}\right\}$. If $\rho$ is a successor ordinal, the sign- expansion of $y_{\beta}$ followed by a plus is an initial subsequence of the sign-expansion of $y$ which in turn is an initial subsequence of the sign-expansion of $y_{\alpha}$ where $y_{\alpha}$ is one of the exponents in $\sum_{\alpha<\beta} \omega^{y_{\alpha}} \cdot r_{\alpha}$, and so the reduced sign-expansion of $y$ consists of the pluses in the sign- expansion of $y_{\beta}$ followed by a single plus. In addition, if $\rho$ is an infinite limit ordinal, then the sign-expansion of $y_{\beta}$ followed by a plus is an initial subsequence of the sign-expansions of the $x_{\alpha} \mathrm{s}$, the union of the latter of which constitutes the signexpansion of $y$, which also implies that the reduced sign-expansion of $y$ consists of the pluses in the sign-expansion of $y_{\beta}$ followed by a single plus. But then in both cases the reduced sign-expansion of $\pm \omega^{y}$ consists of $\omega^{y_{\beta}^{+}+1}$ pluses (minuses) depending on whether \pm is + or - , respectively, which proves (v) and, thereby, the theorem.

\section{A Illustrations of Theorems 3.11, 3.12, 3.14, 3.15, 3.16, and 3.18}

Illustrations of Theorem 3.11(i)

$$
\left(\frac{1}{\omega}\right)^{\mathcal{L}}=\frac{1}{2 \omega} \quad\left(\frac{1}{\omega}\right)^{\mathcal{R}}=\frac{2}{\omega}
$$




\section{Illustrations of Theorem 3.11(ii)}

If $y>0$, then $\tau=0$, and so, $\omega^{\tau}=1$. From this it follows that:

$$
(\omega)^{\mathcal{L}}=\omega-1 \quad(\omega)^{\mathcal{R}}=\omega+1
$$

On the other hand, if $y \leq 0$, then $\tau$ is the greatest inverse of an ordinal $<y$, say, $-\alpha$, and so, $\omega^{\tau}=1 / \omega^{\alpha}$. Accordingly,

$$
(\sqrt{2})^{\mathcal{L}}=\sqrt{2}-\frac{1}{\omega} \quad(\sqrt{2})^{\mathcal{R}}=\sqrt{2}+\frac{1}{\omega}
$$

\section{Illustration of Theorem $\mathbf{3 . 1 2}$}

$$
\left(\sum_{\alpha<\omega} \omega^{\frac{1}{2^{\alpha}}}\right)^{\mathcal{L}}=\sum_{\alpha<\omega} \omega^{\frac{1}{2^{\alpha}}}-1 \quad\left(\sum_{\alpha<\omega} \omega^{\frac{1}{2^{\alpha}}}\right)^{\mathcal{R}}=\sum_{\alpha<\omega} \omega^{\frac{1}{2^{\alpha}}}+1
$$

\section{Illustration of Theorem 3.14}

$$
\text { If } x=\left(\sum_{\alpha<\sigma} \omega^{\frac{1}{2^{\alpha}}}\right)_{\sigma<\omega}, \text { then } \sigma(x)=\sum_{\alpha<\omega} \omega^{\frac{1}{2^{\alpha}}}
$$

\section{Illustration of Theorem 3.15(i)}

$$
\sigma\left(\left(\omega \cdot \frac{1}{2}-\omega^{\frac{1}{2^{n}}}\right)_{n<\omega}\right)=\omega \cdot \frac{1}{2}-\omega^{\frac{1}{\omega}} \quad \sigma\left(\left(\omega \cdot \frac{1}{2}+\omega^{\frac{1}{2^{\alpha}}}\right)_{n<\omega}\right)=\omega \cdot \frac{1}{2}+\omega^{\frac{1}{\omega}}
$$

\section{Illustration of Theorem 3.15(ii)}

$$
\sigma\left(\left(\omega^{\omega} \cdot \frac{1}{2}-\omega^{n}\right)_{n<\omega}\right)=\omega^{\omega} \cdot \frac{1}{4} \quad \sigma\left(\left(\omega^{\omega} \cdot \frac{1}{2}+\omega^{n}\right)_{n<\omega}\right)=\omega^{\omega} \cdot \frac{3}{4}
$$

\section{Illustration of Theorem 3.15(iii)}

$$
\begin{aligned}
& \sigma\left(\left(\omega^{\omega} \cdot \frac{1}{3}-\omega^{n}\right)_{n<\omega}\right)=\omega^{\omega} \cdot \frac{1}{3}-\omega^{\omega-1} \\
& \sigma\left(\left(\omega^{\omega} \cdot \frac{1}{3}+\omega^{n}\right)_{n<\omega}\right)=\omega^{\omega} \cdot \frac{1}{3}+\omega^{\omega-1}
\end{aligned}
$$

\section{Illustration of Theorem 3.16(i)}

$$
\begin{aligned}
& \sigma\left(\left(\omega^{3} \cdot r_{n}\right)_{n<\omega}\right)=\omega^{3} \cdot \frac{1}{3}, \text { if } \sigma\left(\left(r_{n}\right)_{n<\omega}\right)=\frac{1}{3}, \text { i.e. if } \\
& r_{0}=0,\left(r_{1}=1, r_{2}=\frac{1}{2}\right), r_{3}=\frac{1}{4}, r_{4}=\frac{3}{8}, r_{5}=\frac{5}{16}, r_{6}=\frac{11}{32}, \ldots 6
\end{aligned}
$$




\section{Illustration of Theorem 3.16(ii)}

$$
\begin{aligned}
& \sigma\left(\left(\omega^{3} . r_{n}\right)_{n<\omega}\right)=\omega^{3} \cdot \frac{1}{4}+\omega^{\frac{5}{2}}, \text { if } r_{i}=\frac{1}{4}, \text { in which case, } \\
& r_{i+1}=\frac{3}{8}, r_{i+2}=\frac{r_{i}+r_{i+1}}{2}=\frac{5}{16}, \ldots, r_{i+m}=\frac{r_{i}+r_{i+(m-1)}}{2}, \ldots
\end{aligned}
$$

\section{Illustration of Theorem 3.16(iii)}

$$
\begin{aligned}
& \sigma\left(\left(\omega^{3} \cdot r_{n}\right)_{n<\omega}\right)=\omega^{3} \cdot \frac{1}{4}-\omega^{\frac{5}{2}}, \text { if } r_{i}=\frac{1}{4}, \text { in which case, } \\
& r_{i+1}=\frac{1}{8}, r_{i+2}=\frac{r_{i}+r_{i+1}}{2}=\frac{3}{16}, \ldots, r_{i+m}=\frac{r_{i}+r_{i+(m-1)}}{2}, \ldots
\end{aligned}
$$

\section{Illustration of Theorem 3.18((i)}

$$
\sigma\left(\left(\omega^{2} \cdot \frac{3}{4}-\omega^{0} \cdot n\right)_{n<\omega}\right)=\omega^{2} \cdot \frac{3}{4}-\omega \quad \sigma\left(\left(\omega^{2} \cdot \frac{3}{4}+\omega^{0} \cdot n\right)_{n<\omega}\right)=\omega^{2} \cdot \frac{3}{4}+\omega
$$

\section{Illustration of Theorem 3.18(ii)}

$$
\sigma\left(\left(\omega^{2} \cdot \frac{1}{2}-\omega^{1} \cdot n\right)_{n<\omega}\right)=\omega^{2} \cdot \frac{1}{4} \quad \sigma\left(\left(\omega^{2} \cdot \frac{1}{2}+\omega^{1} \cdot n\right)_{n<\omega}\right)=\omega^{2} \cdot \frac{3}{4}
$$

\section{Illustration of Theorem 3.18(iii)}

$$
\sigma\left(\left(\omega^{2} \cdot \frac{1}{3}-\omega^{1} \cdot n\right)_{n<\omega}\right)=\omega^{2} \cdot \frac{1}{3}-\omega^{\frac{3}{2}} \quad \sigma\left(\left(\omega^{2} \cdot \frac{1}{3}+\omega^{1} \cdot n\right)_{n<\omega}\right)=\omega^{2} \cdot \frac{1}{3}+\omega^{\frac{3}{2}}
$$

\section{Illustration of Theorem 3.18(iv)}

$$
\begin{aligned}
& \sigma\left(\left(\omega^{\frac{3}{4}} \cdot \sqrt{2}-\omega^{0} \cdot n\right)_{n<\omega}\right)=\omega^{\frac{3}{4}} \cdot \sqrt{2}-\omega^{\frac{1}{2}} \\
& \sigma\left(\left(\omega^{\frac{3}{4}} \cdot \sqrt{2}+\omega^{0} \cdot n\right)_{n<\omega}\right)=\omega^{\frac{3}{4}} \cdot \sqrt{2}+\omega^{\frac{1}{2}}
\end{aligned}
$$

\footnotetext{
${ }^{6}$ The rule, due to Elwyn Berlekamp [4, page 31], for obtaining the value of a nondyadic positive real number $r$ from its sign-expansion is this: if $r=n+a$, where $n$ and $a$ are the integer part and fractional part of $r$, respectively, then the sign-expansion of $r$ consists of $n+$ signs followed by signs +- , which get bracketed, followed by the ordinary binary expansion of $a$ when 1 is written for + and 0 is written for - . In particular, $\frac{1}{3}$ is the surreal number having the sign- expansion $(+-)-+$ where the underlining indicates the finite sequence of signs that indefinitely recurs. Accordingly, beginning with 0 and inserting the immediate successor on the right (left) of the last term in the sequence obtained thus far whenever $\mathrm{a}+(-)$ occurs in the sign-expansion of $\frac{1}{3}$ results in the sequence specified in the above illustration of Theorem 3.16(i). The individual terms of the sequence can be obtained either by appealing to the rule given in Proposition 3.2 or by appealing to the rule due to Berlekamp (based on sign-expansions) in the citation specified above.
} 


\section{Illustration of Theorem $3.18(v)$}

$$
\begin{aligned}
& \sigma\left(\left(\sum_{\alpha<\omega} \omega^{1+\frac{1}{2^{\alpha}}}-\omega . n\right)_{n<\omega}\right)=\sum_{\alpha<\omega} \omega^{1+\frac{1}{2^{\alpha}}}-\omega^{1+\frac{1}{\omega}} \\
& \sigma\left(\left(\sum_{\alpha<\omega} \omega^{1+\frac{1}{2^{\alpha}}}+\omega . n\right)_{n<\omega}\right)=\sum_{\alpha<\omega} \omega^{1+\frac{1}{2^{\alpha}}}+\omega^{1+\frac{1}{\omega}}
\end{aligned}
$$

\section{References}

[1] N. Alling, Foundations of Analysis Over Surreal Number Fields, North-Holland Publishing Company, Amsterdam, 1987.

[2] N. Alling and P. Ehrlich, Sections 4.02 and 4.03 of [1].

[3] N. Alling and P. Ehrlich, An alternative construction of Conway's surreal numbers, C. R. Math. Rep. Acad. Sci. Canada VIII (1986), 241-46.

[4] J. H. Conway, On Numbers and Games, Academic Press, London, 1976; Second Edition, A K Peters, Ltd., Natick, Massachusetts, 2001.

[5] F. R. Drake, Set Theory; an Introduction to Large Cardinals, North-Holland Publishing Company, Amsterdam, 1974.

[6] L. van den Dries and P. Ehrlich, Fields of surreal numbers and exponentiation, Fund. Math. 167 (2001), 173-188; doi:10.4064/fm167-2-3; erratum, ibid. 168 (2001), 295297; doi: 10.4064/fm168-3-5.

[7] P. Ehrlich, An alternative construction of Conway's ordered field No, Algebra Universalis 25 (1988), 7-16; doi:10.1007/BF01229956; errata, ibid. 25 (1988), 233.

[8] P. Ehrlich, Absolutely saturated models, Fund. Math. 133 (1989), 39-46.

[9] P. Ehrlich, Universally extending arithmetic continua, in Hourya Sinaceur and JeanMichel Salanskis (eds.), Le Labyrinthe du Continu, Colloque de Cerisy, Springer-Verlag France, Paris, 1992, 168-177.

[10] P. Ehrlich, All numbers great and small, in Real Numbers, Generalizations of the Reals, and Theories of Continua, Philip Ehrlich (editor), Kluwer Academic Publishers, Dordrecht, Netherlands, 1994, 239-258

[11] P. Ehrlich, Number systems with simplicity hierarchies: a generalization of Conway's theory of surreal numbers, J. Symbolic Logic 66 (2001), 1231-1258; doi: 10.2307/2695104; corrigendum, ibid. 70 (2005), 1022.

[12] P. Ehrlich, Surreal numbers: an alternative construction (Abstract), Bull. Symbolic Logic 8 (2002), 448.

[13] P. Ehrlich, The absolute arithmetic continuum and the unification of all numbers great and small, Bull. Symbolic Logic, to appear. 
[14] A. Fornasiero, Embedding Henselian fields into power series, J. Algebra 304 (2006), 112-156; doi:10.1016/j.jalgebra.2006.06.037.

[15] H. Gonshor, An Introduction to the Theory of Surreal Numbers, Cambridge University Press, Cambridge, 1986; doi:10.1017/CBO9780511629143.

[16] E. Mendelson, An Introduction to Mathematical Logic, fifth ed., CRC Press, Boca Raton, 2010.

Department of Philosophy, Ohio University, Athens, OH 45701, USA

ehrlich@ohio.edu

http://oak.cats.ohiou.edu/ ehrlich/

Received: 30 June 2010 Revised: 15 November 2010 